\title{
Continuum Theory of Active Phase Separation in Cellular Aggregates
}

\author{
Hui-Shun Kuan $\odot^{1,2,3}$ Wolfram Pönisch ${ }^{2,4,5}$ Frank Jülicher®, ${ }^{2,6,7}$ and Vasily Zaburdaev ${ }^{1,2,3, *}$ \\ ${ }^{1}$ Department of Biology, Friedrich-Alexander-Universität Erlangen-Nürnberg, 91058 Erlangen, Germany \\ ${ }^{2}$ Max Planck Institute for the Physics of Complex Systems, 01187 Dresden, Germany \\ ${ }^{3}$ Max Planck Zentrum für Physik und Medizin, 91058 Erlangen, Germany \\ ${ }^{4}$ MRC Laboratory for Molecular Cell Biology, University College London, WC1E 6BT London, United Kingdom \\ ${ }^{5}$ Department of Physiology, Development and Neuroscience, University of Cambridge, CB2 3DY Cambridge, United Kingdom \\ ${ }^{6}$ Center for Systems Biology Dresden, 01307 Dresden, Germany \\ ${ }^{7}$ Cluster of Excellence Physics of Life, Technische Universität Dresden, 01307 Dresden, Germany
}

(Received 31 March 2020; accepted 29 November 2020; published 4 January 2021)

\begin{abstract}
Dense cellular aggregates are common in biology, ranging from bacterial biofilms to organoids, cell spheroids, and tumors. Their dynamics, driven by intercellular forces, is intrinsically out of equilibrium. Motivated by bacterial colonies as a model system, we present a continuum theory to study dense, active, cellular aggregates. We describe the process of aggregate formation as an active phase separation phenomenon, while the merging of aggregates is rationalized as a coalescence of viscoelastic droplets where the key timescales are linked to the turnover of the active force. Our theory provides a general framework for studying the rheology and nonequilibrium dynamics of dense cellular aggregates.
\end{abstract}

DOI: $10.1103 /$ PhysRevLett.126.018102

Complex biological aggregates, such as bacterial colonies, organoids, cell spheroids, and tumors, are formed by thousands to millions of individual interacting cells. Studies of dense cellular aggregates provide a bridge between tissues and organs to the level of single cells with implications in various biological contexts. Examples include wound healing [1,2], tissue spreading [3-6], tumor growth and treatment $[7,8]$, and formation of biofilms or organoids [8-12]. As the interactions between cells are associated with chemical energy being typically transformed to mechanical work, cellular aggregates are intrinsically out-of-equilibrium (active) systems [13] and behave differently compared to normal viscous liquid droplets [14-18].

A prototypical example of active multicellular aggregates are the microcolonies of Neisseria gonorrhoeae $(N$. gonorrhoeae) bacteria. Microcolonies forming on human epithelial tissue are the infectious units of the second most common sexually transmitted disease gonorrhea. These, as well as numerous other bacteria species, use thin and long retractable filaments called type IV pili to interact with their environment and with each other [19,20] [see Figs. 1(a)-1(c)]. Cycles of pili growth, attachment, retraction, and detachment drive cell motility on surfaces and the

Published by the American Physical Society under the terms of the Creative Commons Attribution 4.0 International license. Further distribution of this work must maintain attribution to the author(s) and the published article's title, journal citation, and DOI. Open access publication funded by the Max Planck Society. process of colony formation. Forces of contracting and constantly remodeling pili networks create a densely packed colony [see Fig. 1(b)] and, together with excluded volume interactions, form an active viscoelastic material.

Multiscale computer simulations have played an important role in biophysical studies of $N$. gonorrhoeae colonies [21-23]. Coarse grained continuum approaches, such as hydrodynamic theories of active matter, have provided powerful concepts to capture large scale emergent behaviors in active systems [24-28]. In biological systems, important insights can often be gained by starting from more specific microscopic models that capture key features of the system of interest. The resulting continuum description is less generic and can provide a deeper understanding of the essential, biologically relevant processes governing the system. Here, starting from microscopic, pili-mediated interactions, we derive the continuum theory of dense active multicellular systems. We show how the turnover of active forces determines the viscoelastic behavior of aggregates. The developed theory rationalizes the formation of N. gonorrhoeae colonies as phase separation and highlights the role of viscoelasticity during the complex process of colonies merging. Thus, we are enabled to, theoretically, fully understand the rheological behavior of multicellular aggregates.

Pili-mediated forces.-Pili-mediated intercellular interactions of bacteria are well studied and serve as an important model example for active cellular force generation. $N$. gonorrhoeae bacteria have multiple $(\sim 10-20)$ pili isotropically distributed over the cell body [see Fig. 1(c)] [29-31]. Statistics of pili length is well captured by an 

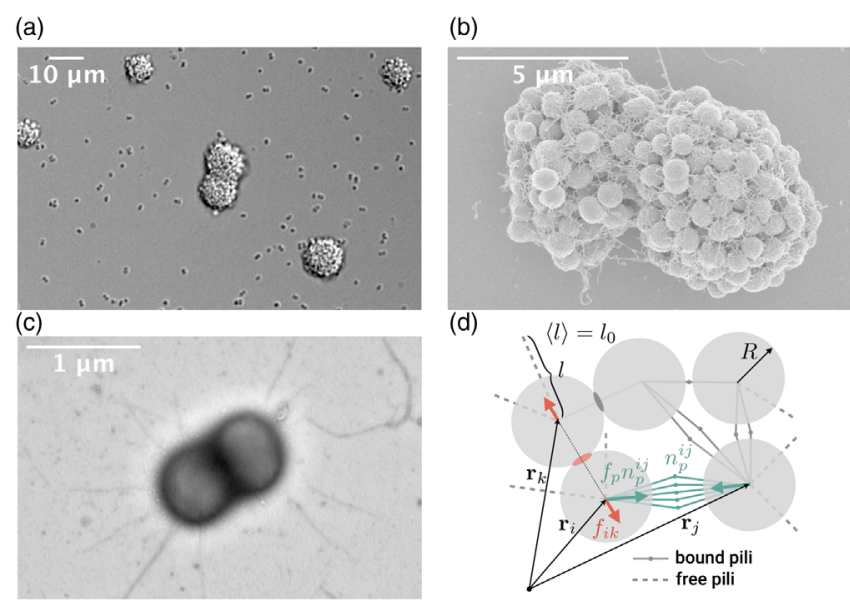

FIG. 1. Bacterial colonies and interactions. (a) N. gonorrhoeae aggregates formed on a solid substrate. (b) Two merging aggregates and pili network. (c) A single cell and its pili. The images are from (a) optical, (b) electron, and (c) transmission electron microscopy. (d) Microscopic forces acting on the cells. Cells are represented as disks of radius $R$. Solid and dashed lines denote pairs of bound and free pili, respectively. Pili length $l$ is exponentially distributed with the mean $l_{0}$. Cells $i$ and $j$ have $n_{p}^{i j}$ bound pili pairs between them. Each pair generates a force dipole of strength $f_{p}$. Thus, $f_{p} n_{p}^{i j}$ is the magnitude of the force acting from cell $j$ on cell $i$ due to the pili, while the green arrow shows its direction. $f_{i k}$ and red arrows show the magnitude and the direction of the steric repulsive force.

exponential distribution with a mean of $l_{0} \sim 1-2 \mu \mathrm{m}$ (diameter of the cell is $\sim 1 \mu \mathrm{m}$ ) [29]. Pili may attach to a substrate or to pili of other $N$. gonorrhoeae bacteria (but not to their cell membrane) [21]. Pili grow (retract) by a process of (de)polymerization driven by specific motor protein complexes [19,30,32-35]. Retraction of an attached pilus generates the pulling force of up to $f_{p} \approx 180 \mathrm{pN}$ $[33,36,37]$. If even a greater stretching force is applied to a pilus, it may extend via a conformational change without detaching [38]. Detachment happens stochastically in a load dependent manner [37]. These key pili characteristics are, in some instances with simplifications, introduced in the microscopic model below.

Cell-based model of aggregate dynamics.-In a simplified two-dimensional setting, cells are represented as disks with the radius $R$ [see Fig. 1(d)]. Pili are formed stochastically, with isotropic orientation and with an exponential length distribution with average $l_{0}$. Two individual pili of two neighboring cells may bind to each other. Retraction of the pili generates no net force acting on the two cells, but it gives rise to the attractive force dipole of strength $f_{p}$ pulling the cells together [see Fig. 1(d)]. Pili can also bind to the substrate. Pili-substrate interactions can drive bacterial motility and are important for understanding the kinetics of aggregation as has been shown before [39]. Here, we focus on the behavior of dense aggregates and, thus, do not consider effects due to pili-substrate interactions beyond substrate friction [23]. In the overdamped limit, for an individual cell, the pili-pili mediated forces are balanced by excluded volume interactions and substrate friction [see Fig. 1(d)]

$$
f_{p} \sum_{j} \hat{\mathbf{i}}_{j i} n_{p}^{j i}+\sum_{j} \mathbf{f}_{j i}-\xi \mathbf{v}_{i}=0
$$

where $\mathbf{v}_{i}$ is the velocity of cell $i$ on the substrate and $\xi$ is a friction coefficient. The steric repulsion force exerted by cell $j$ on cell $i$ is denoted $\mathbf{f}_{j i}$. The unit vector $\hat{\mathbf{I}}_{j i}=\mathbf{l}_{j i} / l_{j i}$ shows the direction of the pili-pili mediated force exerted by cell $j$ on cell $i$, where $\mathbf{l}_{j i}=\mathbf{r}_{j}-\mathbf{r}_{i}$ is the vector pointing from cell $i$ at the position $\mathbf{r}_{i}$ to cell $j$ at the position $\mathbf{r}_{j}$, and $l_{j i}$ is the corresponding distance. The pili-pili mediated force between cells is proportional to the number of bound pili pairs between cells $i$ and $j$, denoted $n_{p}^{j i}$ [see Fig. 1(d)]. Note that $n_{p}^{j i}$ is changing with time. We consider formation of bound pili pairs with a binding constant $k_{\text {on }}$ and assume that the detachment happens with a constant rate $k_{\text {off }}$. Because the steady-state pili length distribution is exponential, the number of bound pili pairs obeys (for details, see [40])

$$
\frac{d}{d t} n_{p}^{j i}=\frac{k_{\mathrm{on}} e^{-\frac{l_{j i}}{l_{0}}}}{2 \pi l_{0}^{2}}-k_{\mathrm{off}} n_{p}^{j i} .
$$

Continuum limit.-At the microscopic level, there are two key features signifying nonequilibrium nature of the system and distinguishing it from previous models of active particle systems [45-48]. First, the network of bound pili leads to an elasticlike response to deformation at time scales smaller than the pili detachment time. At larger time scales, pili can rearrange, leading to stress relaxation and fluidlike material response. Pili turnover and force generation are also responsible for breaking the detailed balance. Second, the pili-pili mediated forces acting as attractive force dipoles are balanced by steric repulsion forces allowing us to consider the regime of dense cellular aggregates. These two features, as well as the details of pili interactions, are captured by the resulting continuum equations. Now, we use the microscopic force balance equation together with pili turnover to obtain dynamic equations for coarse-grained cell number density $n$ and force balance (the coarse-graining procedure is described in [40])

$$
\partial_{t} n=-\nabla \cdot(\mathbf{v} n), \quad \xi n \mathbf{v}=\nabla \cdot \underline{\underline{\sigma}}^{a}+\nabla \cdot \underline{\underline{\sigma}}^{p} .
$$

Here, $\mathbf{v}$ is the cell velocity field, and $\underline{\sigma}^{a, p}$ are the active and passive stress tensors, respectively. The expression for the passive stress incorporates effects from short range repulsive interactions. In the continuum, we use the expression

$$
\underline{\sigma}^{p}=-\frac{E v_{c} n}{1-v_{c} n} \underline{\underline{I}},
$$


where $v_{c}=\pi R^{2}$ is the area of a single cell, $E$ is the bulk elastic modulus, and $I$ is the identity matrix. This, in fact, is the van der Waals gas law for pressure without attractive interaction, and is one of the simplest forms for describing the excluded volume effects.

Because of the intrinsic nature of pili-pili interaction as force dipoles [25], the first nontrivial term of the active stress has nematic symmetry $[25,49]$ and can be written as $\underline{\underline{\sigma}}^{a}=f_{p} \underline{\underline{N}}$, where

$$
\underline{\underline{N}}=\sum_{i \neq j}\left\langle\mathbf{l}_{j i} \hat{\mathbf{l}}_{j i} n_{p}^{i j} \delta\left(\mathbf{r}-\frac{\mathbf{r}_{i}+\mathbf{r}_{j}}{2}\right)\right\rangle
$$

is a nematic tensor which characterizes the amount of the bound pili pairs and their axis of anisotropy (see [40]).

Phase separation.-The continuum theory allows for a phase separation regime where attractive pili-pili forces drive the formation of dense aggregates from an initially homogenous distribution of cells [see Figs. 2(a) and 2(b),
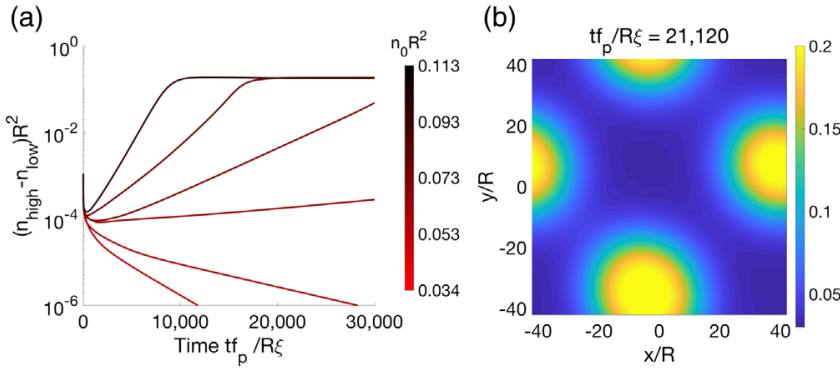

(c)

(d)
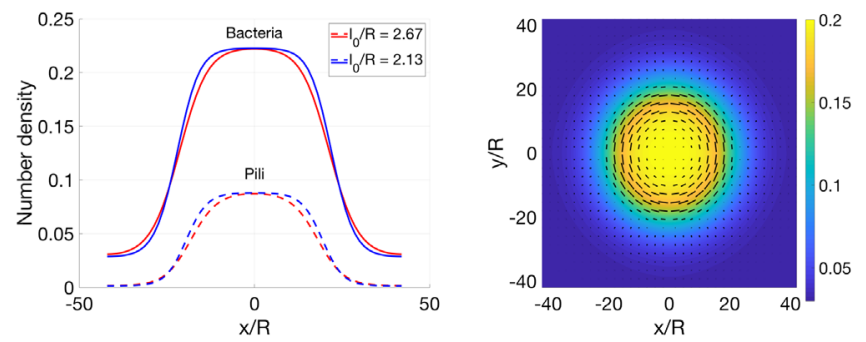

FIG. 2. Aggregate formation as a phase separation phenomenon. (a) Difference of high and low cell number density phases as a function of time. The critical initial density of the onset of the phase separation is at $n_{c} R^{2} \simeq 0.049$ for $k_{\text {on }} / k_{\text {off }} R^{2}=1.78$, the initial density fluctuation level of 0.001 , and pili length is set $l_{0} / R=2.67$ [for all panels except (c)]. (b) The snapshot of the density after the onset of the phase separation [initial density is $n_{0} R^{2}=0.079$ as in the second line from top of panel (a), and $\left.f_{p} / \pi R E=6\right]$. (c) The cell number density (solid curve) and total number of bound pili density (dashed curve) of stable aggregates for two different pili lengths. The bulk elastic moduli are taken as $f_{p} / \pi R E=6$ (smaller plateau) and $f_{p} / \pi R E=7.5$ (larger plateau) to provide the same degree of phase separation. The pili length controls the width of the aggregate's boundary $\left(n_{0} R^{2}=0.072\right)$. (d) The nematic tensor $\underline{\underline{N}}-\frac{1}{2} \operatorname{Tr}[\underline{\underline{N}}]$ inside of a stabilized aggregate $\left(n_{0} R^{2}=0.072\right)$. Lines and their length indicate the nematic ordering direction and the order parameter, respectively. movie S1]. Here, we take advantage of the adiabatic approximation assuming that pili turnover, Eq. (2), happens much faster than the motion of cells. The resulting, much simplified equation for cell density, is of the Cahn-Hilliard type $[39,50]$. By applying the linear stability analysis, we can find the critical density $n_{c}$ above which the initially homogeneous state becomes unstable with respect to small density fluctuations [Fig. 2(a) and [40]]

$$
\frac{f_{p} k_{\mathrm{on}} l_{0}}{k_{\mathrm{off}}} n_{c}-\frac{E v_{c}}{\left(1-n_{c} v_{c}\right)^{2}}=0 .
$$

Above the critical density, multiple, similarly sized aggregates emerge [Fig. 2(b)] with a characteristic length scale, which can also be derived from the linear stability analysis. On a longer time scale, coarsening dynamics leads to the formation of a single stabilized aggregate, Figs. 2(c) and 2(d). This adiabatic regime also admits an effective free-energy, equilibriumlike description (see [40]).

Dynamic equations.-Now, we discuss the equations describing dynamics of colony formation. The time evolution of the active stress is determined by calculating its time derivative in the coarse-graining procedure. Naturally, it leads to the appearance of higher rank tensors, which we estimate using a standard closure approximation and by introducing the density of the total number of bound pili $N$ (see [40])

$$
\begin{aligned}
\frac{d \sigma_{\alpha \beta}^{a}}{d t}= & f_{p}\left(N_{\alpha \gamma} \partial_{\gamma} v_{\beta}-N_{\beta \alpha} \partial_{\gamma} v_{\gamma}+N_{\beta \gamma} \partial_{\gamma} v_{\alpha}-\frac{N_{\alpha \beta} N_{\epsilon \gamma} \partial_{\epsilon} v_{\gamma}}{N l_{0}}\right) \\
& +4 f_{p} k_{\mathrm{on}} \int d^{2} l \frac{l_{\beta} l_{\alpha}}{l} \frac{e^{-2 l / l_{0}}}{2 \pi l_{0}^{2}} n(\mathbf{r}+\mathbf{l}) n(\mathbf{r}-\mathbf{l}) \\
& -f_{p} k_{\mathrm{off}} N_{\alpha \beta} .
\end{aligned}
$$

Equation (7) describes the stress-strain relation due to cell movement (the first term), the active stress from the newly formed pili pairs (the second term), and the relaxation by the detachment of bound pili (the last term). Together with continuity and force balance equations, Eq. (3), and time evolution for $N$, it constitutes the full system describing the dynamics (see [40]). Coupling of the nematic tensor to cell movement for the finite pili turnover is the source of the broken detailed balance that does not allow for an equilibriumlike description. For linearized dynamic equations, there exists a region in parameter and wave vector space corresponding to complex eigenvalues and, thus, oscillatory behavior. A homogeneous density perturbed by a mode with a suitable wave length will oscillate with a decreasing amplitude, exemplifying a nonequilibrium behavior [40,51].

Viscoelasticity.-Next, we discuss the material properties of colonies mediated by pili forces and controlled by the nematic tensor $N$. The active stress Eq. (7) relaxes at a rate $k_{\text {off }}$, implying viscoelastic material properties. Combined with the definition of the active stress Eq. (5), the relaxation 
dynamics can be rewritten as the Maxwell model with a source term due to newly binding pili

$$
\begin{aligned}
\left(1+\frac{1}{k_{\mathrm{off}}} \frac{d}{d t}\right) \sigma_{\alpha \beta}^{a}= & \frac{\lambda_{\alpha \beta \gamma \epsilon}}{k_{\mathrm{off}}} \partial_{\gamma} v_{\epsilon}+4 f_{p} \frac{k_{\mathrm{on}}}{k_{\mathrm{off}}} \int d^{2} l \frac{l_{\beta} l_{\alpha}}{l} \frac{e^{-2 l / l_{0}}}{2 \pi l_{0}^{2}} \\
& \times n(\mathbf{r}+\mathbf{l}) n(\mathbf{r}-\mathbf{l}),
\end{aligned}
$$

where $\quad \lambda_{\alpha \beta \gamma \epsilon}=f_{p}\left(N_{\alpha \gamma} \delta_{\beta \epsilon}+N_{\beta \gamma} \delta_{\alpha \epsilon}-N_{\alpha \beta} \delta_{\gamma \epsilon}-\left(N_{\alpha \beta} N_{\gamma \epsilon} /\right.\right.$ $\left.\left.N l_{0}\right)\right)$ is an elastic tensor. At short times $\left(t \ll k_{\text {off }}^{-1}, k_{\text {on }}^{-1}\right)$, the active stress behaves elastically. At larger times $\left((d / d t) \sigma_{\alpha \beta}^{a} \simeq 0\right)$, the network of bound pili pairs is remodeled due to the binding or unbinding processes, and the active stress behaves as in a viscous fluid with the viscous tensor $\eta_{\alpha \beta \gamma \epsilon}=\lambda_{\alpha \beta \gamma \epsilon} k_{\text {off }}^{-1}$ (calculated from the ratio between the strain and relaxing stress). The viscoelastic behavior emerges naturally in the continuum description and shows intimate relation to the pili turnover dynamics. Pili-induced elastic moduli and viscosities explicitly depend on the cell number density. At the surface of the colony, the density is lower than in the bulk, and thus, we expect the elastic moduli and the viscosities to be smaller at the surface. Thus, our theory naturally accounts for the experimentally observed effect of heterogeneous cell motility in $N$. gonorrhoeae aggregates [21,23]. This heterogeneity, in turn, effects the merging of colonies, a complex dynamic process and the major mechanism of colony growth considered next.

Coalescence of colonies.-The merging of cellular aggregates [Fig. 3(a)] is reminiscent of a coalescence of liquid droplets [21,52-54]. It can be quantified by following the "liquid (capillary) bridge" height $h(t)$ [inset in top panel of Fig. 3(b)] as a function of time. Overall, we see an interesting behavior which is not captured by a single time scale. Because of the viscoelastic nature of the active stress [see Eq. (6)], at short times $t \ll k_{\text {off }}^{-1}$, the merging is dominated by the cell movements without pili detachment and leads to an elastic response. At large times $t \gg k_{\text {off }}^{-1}$, the bound pili network has enough time to remodel, leading to an effective viscous behavior, and gives rise to a time scale governed by the ratio of the effective viscosity to the average stresses.

For the increasing force $f_{p}$ and decreasing pili detachment rate $k_{\text {off }}$, aggregates tend to merge faster [Fig. 3(b)]. During the late stage of the merging, an additional time scale from the ratio of the effective viscosity and the stress tensor becomes relevant [55-58], which, due to the similar scaling of the effective viscosity, linearly depends on $k_{\text {off }}^{-1}$ [inset in the middle panel of Fig. 3(b)]. By dimensional analysis, we find four nondimensional parameters: $l_{0} / R$, $k_{\mathrm{on}} / k_{\mathrm{off}} R^{2}, f_{p} / \pi R E, f_{p} / R \xi k_{\mathrm{off}}$ and the rescaled time $t f_{p} / R \xi$ which determine the scaling of the system. The first is the ratio of the mean pili length and the cell radius, the second is related to the number of bound pili, and the third is the ratio between the active and excluded volume forces, which together determine the degree of the phase separation [see, also, Eq. (6)]. The fourth parameter is the (a)

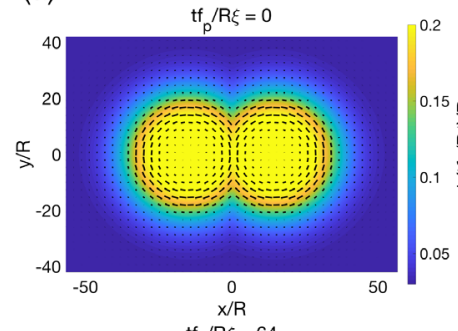

(b)
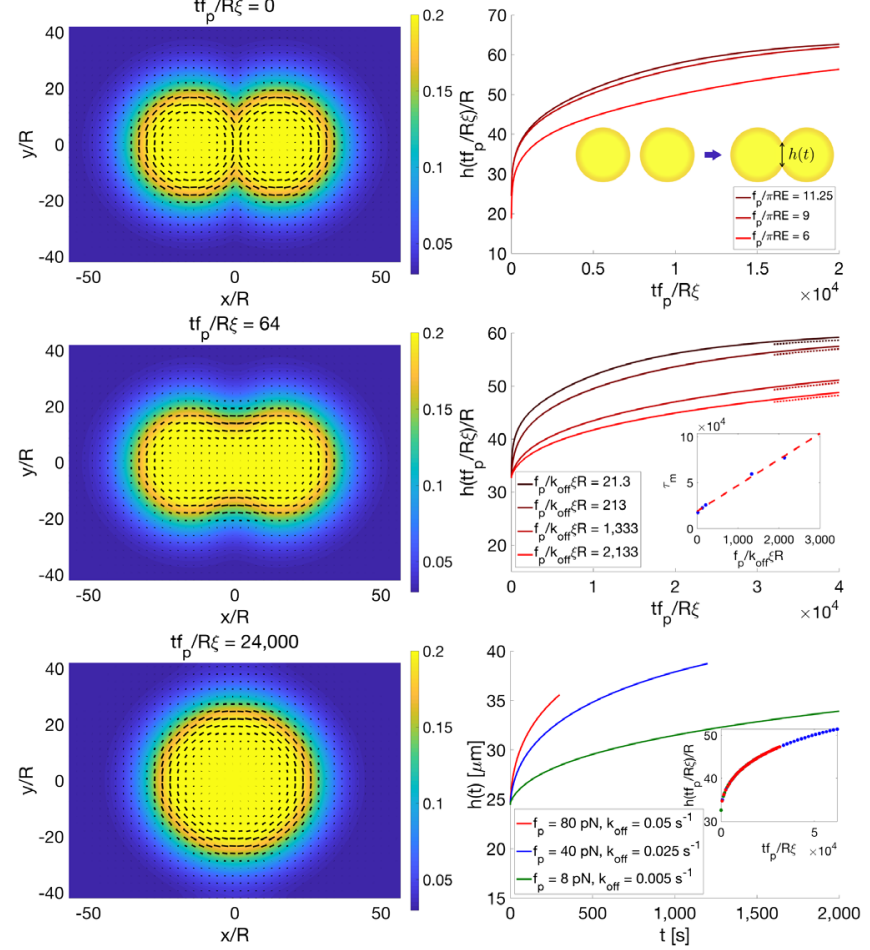

FIG. 3. Coalescence of aggregates. (a) The snapshots of two aggregates merging at different times $\left(k_{\mathrm{on}} \xi / f_{p} R=0.083\right.$, $k_{\text {off }} \xi R / f_{p}=0.047$, and $f_{p} / \pi R E=6$, Movie S2). Lines indicate the magnitude and direction of the nematic ordering as in Fig. 2(d). (b) The bridge height $h / R$ as the function of the rescaled time $t f_{p} / R \xi$. Top: inset shows definition of $h(t)$. The boundary of the aggregate is defined as the level set of midpoint between the low and high density. Changing $f_{p}$, while $k_{\mathrm{on}}$ and $k_{\text {off }}$ are kept constant, is shown with gradient of colors, with darker color corresponding to higher force. Middle: changing $k_{\text {off }}$, where $f_{p} / \pi R E=6$ and $k_{\text {on }} / R^{2} k_{\text {off }}=1.78$ are kept constant to guarantee the same degree of phase separation. The gradient of colors indicates the values of $k_{\text {off }}$, with the darker color corresponding to higher value of $k_{\text {off }}$. The inset shows the asymptotic merging time $\tau_{m}$ (corresponding to the dashed lines in the main plot) which linearly depends on $k_{\mathrm{off}}^{-1}$ due to the effective viscosity. Bottom: changing both $k_{\text {off }}$ and $f_{p}$. The inset shows the master curve after rescaling as discussed in the text. The top curve is close to the parameter regime considered in the previous study [21].

ratio between the time for the cell to move its radius under the pili force and the pili detachment time, which is the only nondimensional parameter which can be varied if the degree of the phase separation is fixed. Figure 3(b) (bottom) confirms these scalings by collapsing the plots for the bridge height as a function of time for different parameters on a single master curve.

Conclusions. - Our work provides a systematic derivation of a continuum theory for studying active phase separation and rheology of dense cellular aggregates. The contribution of pili-mediated forces is incorporated via turnover kinetics. For finite turnover rates, the coupling 
of the nematic tensor to cell motion breaks detailed balance. This potentially allows for steady states with nonzero currents, and leads to damped oscillations when the system is perturbed from the steady state, see [40,51]. For fast pili turnover, the model behaves effectively like an equilibrium system. Interestingly, for finite pili turnover, a steady state, such as a circular droplet, exists, where the macroscopic velocity vanishes and the system does not exhibit nonequilibrium currents. It would be interesting to identify general criteria for which an active system driven by active force dipoles can be captured by an effective equilibrium description. Force generation similar to pili-pili interactions is common in many biological systems, such as interacting cells or active gels [25,59]. The coupling between the active stress and cell motion gives rise to space and time dependent elastic moduli and viscosities, thus, enabling our model to treat inhomogeneous systems, a prerequisite for eventually understanding the morphogenesis of tissues.

We thank C. A. Weber, T. Harmon, J. Löber, S. Saha, L. Hilbert, and R. Seyboldt for useful discussions on the concepts of phase separation. We also thank N. Biais for detailed discussion and providing images for Figs. 1(a)-1(c). This work was supported by Volkswagen foundation "Life?" initiative (H.S. K. and V.Z.). W. P. thanks the Leverhulme Trust and the Herchel Smith Postdoctoral Fellowship Fund.

*vasily.zaburdaev@fau.de

[1] M. T. Armstrong and P. B. Armstrong, J. Cell Sci. 102, 373 (1992), https://jcs.biologists.org/content/102/2/373.

[2] D. Bi, X. Yang, M. C. Marchetti, and M. L. Manning, Phys. Rev. X 6, 021011 (2016).

[3] G. Beaune, T. V. Stirbat, N. Khalifat, O. Cochet-Escartin, S. Garcia, V. V. Gurchenkov, M. P. Murrell, S. Dufour, D. Cuvelier, and F. Brochard-Wyart, Proc. Natl. Acad. Sci. U.S.A. 111, 8055 (2014).

[4] G. Beaune, G. Duclos, N. Khalifat, T. V. Stirbat, D. M. Vignjevic, and F. Brochard-Wyart, Soft Matter 13, 8474 (2017).

[5] S. Douezan, K. Guevorkian, R. Naouar, S. Dufour, D. Cuvelier, and F. Brochard-Wyart, Proc. Natl. Acad. Sci. U.S.A. 108, 7315 (2011).

[6] S. Douezan, J. Dumond, and F. Brochard-Wyart, Soft Matter 8, 4578 (2012).

[7] W. Mueller-Klieser, Journal of cancer research and clinical oncology 113, 101 (1987).

[8] T. Eguchi et al., PLoS One 13, e0191109 (2018).

[9] E. Ben-Jacob, Cohen, Inon, and D. L. Gutnick, Annu. Rev. Microbiol. 52, 779 (1998).

[10] L. R. Johnson, J. Theor. Biol. 251, 24 (2008).

[11] N. Høiby, T. Bjarnsholt, M. Givskov, S. Molin, and O. Ciofu, Int. J. Antimicrob. Agents 35, 322 (2010).

[12] H. Clevers, Cell 165, 1586 (2016).

[13] M. C. Marchetti, Nature (London) 491, 340 (2012).

[14] W. Xi, T. B. Saw, D. Delacour, C. T. Lim, and B. Ladoux, Nat. Rev. Mater. 4, 23 (2019).
[15] C. T. Lim, E. H. Zhou, and S. T. Quek, J. Biomech. 39, 195 (2006).

[16] D. T. Chen, Q. Wen, P. A. Janmey, J. C. Crocker, and A. G. Yodh, Annu. Rev. Condens. Matter Phys. 1, 301 (2010).

[17] M. Gupta, B. R. Sarangi, J. Deschamps, Y. Nematbakhsh, A. Callan-Jones, F. Margadant, R.-M. Mège, C. T. Lim, R. Voituriez, and B. Ladoux, Nat. Commun. 6, 7525 (2015).

[18] D. Mizuno, C. Tardin, C. F. Schmidt, and F. C. MacKintosh, Science 315, 370 (2007).

[19] L. Craig, M. E. Pique, and J. A. Tainer, Nat. Rev. Microbiol. 2, 363 (2004).

[20] J. S. Mattick, Annu. Rev. Microbiol. 56, 289 (2002).

[21] W. Pönisch, C. A. Weber, G. Juckeland, N. Biais, and V. Zaburdaev, New J. Phys. 19, 015003 (2017).

[22] D. Bonazzi, V. Lo Schiavo, S. Machata, I. Djafer-Cherif, P. Nivoit, V. Manriquez, H. Tanimoto, J. Husson, N. Henry, H. Chaté, R. Voituriez, and G. Duménil, Cell 174, 143 (2018).

[23] W. Pönisch, K. B. Eckenrode, K. Alzurqa, H. Nasrollahi, C. Weber, V. Zaburdaev, and N. Biais, Sci. Rep. 8, 16567 (2018).

[24] F. Jülicher, K. Kruse, J. Prost, and J.-F. Joanny, Phys. Rep. 449, 3 (2007).

[25] J. Prost, F. Jülicher, and J.-F. Joanny, Nat. Phys. 11, 111 (2015).

[26] A. C. Callan-Jones and F. Jülicher, New J. Phys. 13, 093027 (2011).

[27] J. Ranft, M. Basan, J. Elgeti, J.-F. Joanny, J. Prost, and F. Julicher, Proc. Natl. Acad. Sci. U.S.A. 107, 20863 (2010).

[28] M. Popović, A. Nandi, M. Merkel, R. Etournay, S. Eaton, F. Jülicher, and G. Salbreux, New J. Phys. 19, 033006 (2017).

[29] C. Holz, D. Opitz, L. Greune, R. Kurre, M. Koomey, M. A. Schmidt, and B. Maier, Phys. Rev. Lett. 104, 178104 (2010).

[30] V. Zaburdaev, N. Biais, M. Schmiedeberg, J. Eriksson, A.B. Jonsson, M. P. Sheetz, and D. A. Weitz, Biophys. J. 107, 1523 (2014).

[31] J. Eriksson, O. S. Eriksson, L. Maudsdotter, O. Palm, J. Engman, T. Sarkissian, H. Aro, M. Wallin, and A.-B. Jonsson, BMC Microbiol. 15, 92 (2015).

[32] R. Marathe, C. Meel, N. C. Schmidt, L. Dewenter, R. Kurre, L. Greune, M. Alexander Schmidt, M. J. Müller, R. Lipowsky, B. Maier, and S. Klumpp, Nat. Commun. 5, 3759 (2014).

[33] J. M. Skerker and H. C. Berg, Proc. Natl. Acad. Sci. U.S.A. 98, 6901 (2001).

[34] B. Maier, Soft Matter 9, 5667 (2013).

[35] L. Craig, K. T. Forest, and B. Maier, Nat. Rev. Microbiol. 17, 429 (2019).

[36] A. J. Merz, M. So, and M. P. Sheetz, Nature (London) 407 , 98 (2000).

[37] B. Maier, L. Potter, M. So, H. S. Seifert, and M. P. Sheetz, Proc. Natl. Acad. Sci. U.S.A. 99, 16012 (2002).

[38] N. Biais, D. L. Higashi, J. Brujić, M. So, and M. P. Sheetz, Proc. Natl. Acad. Sci. U.S.A. 107, 11358 (2010).

[39] C. A. Weber, Y. T. Lin, N. Biais, and V. Zaburdaev, Phys. Rev. E 92, 032704 (2015).

[40] See Supplemental Material at http://link.aps.org/ supplemental/10.1103/PhysRevLett.126.018102 for detailed derivations, which includes Refs. [41-44]. 
[41] B. Maier and G. C. L. Wong, Trends Microbiol. 23, 775 (2015).

[42] M. Doi, J. Polym. Sci. 19, 229 (1981).

[43] W. H. Press, S. A. Teukolsky, W. T. Vetterling, and B.P. Flannery, Numerical Recipes 3rd Edition: The Art of Scientific Computing, 3rd ed. (Cambridge University Press, New York, 2007).

[44] G. R. Dennis, J. J. Hope, and M. T. Johnsson, Comput. Phys. Commun. 184, 201 (2013).

[45] Y. Fily and M. C. Marchetti, Phys. Rev. Lett. 108, 235702 (2012).

[46] S. Henkes, Y. Fily, and M. C. Marchetti, Phys. Rev. E 84, 040301(R) (2011).

[47] E. Bertin, M. Droz, and G. Grégoire, Phys. Rev. E 74, 022101 (2006).

[48] J. Palacci, S. Sacanna, A. P. Steinberg, D. J. Pine, and P. M. Chaikin, Science 339, 936 (2013).

[49] Y. Hatwalne, S. Ramaswamy, M. Rao, and R. A. Simha, Phys. Rev. Lett. 92, 118101 (2004).

[50] A. J. Bray, Adv. Phys. 43, 357 (1994).
[51] S. Saha, J. Agudo-Canalejo, and R. Golestanian, Phys. Rev. X 10, 041009 (2020).

[52] K. Jakab, B. Damon, F. Marga, O. Doaga, V. Mironov, I. Kosztin, R. Markwald, and G. Forgacs, Dev. Dyn. 237, 2438 (2008).

[53] A. Welker, T. Cronenberg, R. Zöllner, C. Meel, K. Siewering, N. Bender, M. Hennes, E. R. Oldewurtel, and B. Maier, Phys. Rev. Lett. 121, 118102 (2018).

[54] L. Oswald, S. Grosser, D. M. Smith, and J. A. Käs, J. Phys. D 50, 483001 (2017).

[55] E. Flenner, L. Janosi, B. Barz, A. Neagu, G. Forgacs, and I. Kosztin, Phys. Rev. E 85, 031907 (2012).

[56] E. D. Siggia, Phys. Rev. A 20, 595 (1979).

[57] J. Eggers, J. R. Lister, and H. A. Stone, J. Fluid Mech. 401, 293 (1999).

[58] C. T. Bellehumeur, M. Kontopoulou, and J. Vlachopoulos, Rheol. Acta 37, 270 (1998).

[59] A. Mietke, F. Jülicher, and I. F. Sbalzarini, Proc. Natl. Acad. Sci. U.S.A. 116, 29 (2019). 